\title{
Setting a Protocol for Identification and Detecting the Prevalence of Candida auris in Tertiary Egyptian Hospitals Using the CDC Steps
}

\author{
Sahar Mohammed Khairat, Mervat Gaber Anany, Maryam Mostafa Ashmawy $\mathbb{D}$, Amira Farouk Ahmed Hussein* (D) \\ Department of Clinical and Chemical Pathology, Faculty of Medicine, Cairo University, Giza, Egypt
}

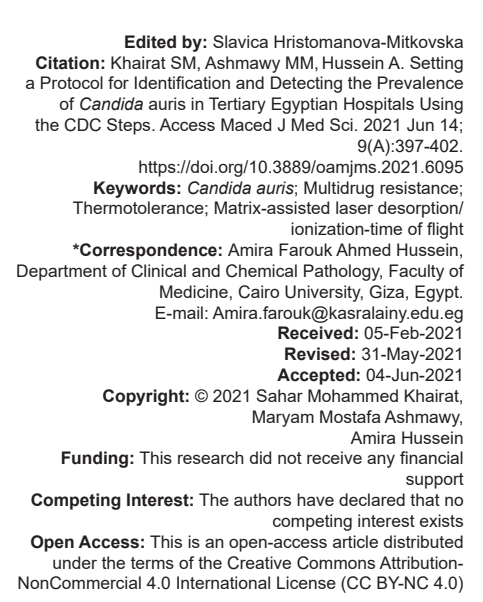

Introduction

Fungal infections are becoming more widely accepted as a global health problem. A fungal infection affects approximately 1.7 billion people worldwide, the majority of which are superficial infections of the skin and mucosa [1]. Candida species are the most common cause of nosocomial fungal infections and the fourth most common source of hospitalacquired infections. Globally, Candida species cause approximately 400,000 bloodstream infections per year, with mortality rates exceeding $40 \%$. Candida albicans is the most common Candida species, although nonalbicans species such as Candida tropicalis, Candida parapsilosis, and Candida glabrata have become more common due to long-term use and reduced antifungal treatment choices [2].

Candida auris, one of non-albicans species, is an emerging invasive multidrug-resistant fungal infection. It has been reported from 42 countries and presents a serious global health threat [3]. It causes infections in hospital settings around the world. Such hospital-acquired infections have proven to be very difficult to diagnose and treat. Individuals, exposed to this pathogen, are at a high risk for systemic infections resulting in high mortality rates [4]. It has been reported as a major rapidly emerged cause of candidemia worldwide, surpassing the number of cases caused by C. glabrata and C. tropicalis in South Africa [5].

C. auris is difficult to be identified with standard phenotypic laboratory methods, and only specific technology such as matrix-assisted laser desorption ionization-time of flight (MALDI-TOF) mass spectrometry and DNA sequencing can distinguish it effectively from other yeasts. Misidentification may lead to inappropriate management and spread of infection in health-care settings [6].

Furthermore, there is a significant genetic heterogeneity among $C$. auris strains from different parts of the world. C. auris has been divided into five distinct clades using whole-genome sequencing-based phylogenetics [7]. 
For this reason, it is important to set a protocol to rapidly identify it in hospitalized patients; thus, health-care facilities can take special precautions and implementing effective infection control measures to stop its spread especially because some commonly used health-care disinfectants are not sufficiently effective against $C$. auris leads to its persistence in health-care environments for several weeks [8].

However, even as knowledge about $C$. auris grows, notable gaps remain in the understanding of Candida auris emergence, spread, and resistance [9]. Especially from countries such as Qatar, Jordan, and Egypt [10].

The purpose of this study is to set a protocol and detect the prevalence of $C$. auris in tertiary Egyptian hospitals following the center of disease and control (CDC) in detection [11].

\section{Methods}

The study was conducted on all samples sent to Cairo University Hospitals with positive Candida growth, during the period from February 2018 to December 2019.

\section{Clinical data}

Patients' wards and types of samples of candida non-albicans were collected from the associated routine request form of patients' samples delivered to the laboratories. The research protocol was approved by the Research Ethical Committee and following the ethical rules of Faculty of medicine Cairo University Hospitals under ID I-251015.

\section{Selection of samples and isolates}

Samples shown growth for Candida on blood chocolate, or MacConkey agar plates were collected, subcultured on Sabouraud dextrose agar for isolation of Candida (Biomerieux, France REF, 43 651) [12].

Isolates either pathogenic or colonizers were collected as $C$. auris could be detected as pathogen or as a colonizer [13].

\section{species level \\ Primary identification of isolates to}

Germ tube method was used to categorize candida isolates to $C$. albicans and Candida species. The method is as following; incubating some Candida colonies in $400 \mathrm{ul}$ of human serum for $3 \mathrm{~h}$ in $37^{\circ} \mathrm{C}$ then examined with a wet $\mathrm{KOH}$ films for filamentous outgrowth extending from yeast cells. It is positive for $C$. albicans and Candida dubliniensis and negative for other species [14].

\section{Confirmation of isolates identification by} chromogenic agar medium

Further species identification to C. albicans, C. tropicalis, Candida krusei, and other species was performed using chromogenic media (Oxoid England, Ref CM100) [15]. Agar was prepared and result interpreted according to the manufacture guidelines in Table 1.

Table 1: Identification of candida based on substrate color change in chromogenic media

\begin{tabular}{ll}
\hline Candida type & Typical colony color appearance \\
\hline C. tropicalis & Dark blue \\
C. albicans & Green \\
C. krusei & Dry, irregular pink-brown \\
C. glabrata, C. kefyr, C. parapsilosis, C. lusitaniae & Beige/yellow/ brown \\
\hline C. tropicalis: Candida tropicalis, C. albicans: Candida albicans, C. krusei: Candida krusei, C. glabrata: \\
Candida glabrata, C. kefyr. Candida kefyr, C. parapsilosis: Candida parapsilosis, C. lusitaniae: Candida. \\
lusitaniae.
\end{tabular}

\section{Thermotolerance to detect suspected isolates}

Isolates that appeared non-albicans on chromogenic medium were further subjected to thermotolerance. This was done by subculturing isolates on chromogenic medium as discussed before, but the plates were incubated at $40-42^{\circ} \mathrm{C}$ for 24-48 h. Only C. albicans and few species including C. auris can grow at $42^{\circ} \mathrm{C}$. C. albicans was used as a control [11].

\section{C. auris}

\section{MALDI-TOF for definite diagnosis of}

Isolates that appeared non-albicans and grew on $42^{\circ} \mathrm{C}$ were subjected to bioMérieux VITEK (MALDITOF) MS research use only libraries (with Saramis Ver 4.14 database and Saccharomycetaceae update) according to CDC [16].

\section{Resistance pattern of MALDI-TOF identified isolates}

Resistance in identified candida species was tested using three antifungal $E$ tests, amphotericin $B$, caspofungin, and fluconazole (Biomerieux). The MIC results were interpreted according to CLSI guidelines M60, 2017 [17].

The European Committee on Antimicrobial Susceptibility Testing was used for interpretation of amphotericin B because it is not yet identified by CLSI [18].

\section{Quality control strains}

C. parapsilosis ATCC 22019 was used to ensure the quality of antifungal $E$ tests used in detection of the antifungal resistance pattern [16]. 


\section{Statistical analysis}

Statistical calculations were done using computer programs Statistical Package for the Social Sciences (SPSS Inc., Chicago, IL, USA) version 15 for Microsoft Windows. Percentage from total analysis was used using the following equation part/whole $\times 100$.

\section{Results}

This is a cross-sectional study that was conducted on 400 Candida isolates collected from different samples sent to microbiology laboratories of Cairo University Hospitals during the period from February 2018 to December 2019.

All Candida isolates were subjected to germ tube and cultured on chromogenic agar medium which were classified as follows; 227 (56.75\%) C. albicans and 180 (43.25\%) were non-albicans Candida. Candida non-albicans was further classified by chromogenic agar as following; 25 (13.8\%) were C. tropicalis, $43(23.8 \%)$ were C. krusei, and 112 (62.2\%) were other Candida species (C. glabrata, Candida kefyr, C. parapsilosis, and Candida lusitaniae), as shown in Table 2.

Table 2: Classification of candida non-albicans by chromagar

\begin{tabular}{|c|c|c|c|c|c|c|c|c|}
\hline \multicolumn{2}{|c|}{ Candida species } & \multirow{2}{*}{\multicolumn{2}{|c|}{ Number }} & \multirow{2}{*}{\multicolumn{2}{|c|}{ Percentage }} & \multicolumn{3}{|c|}{ Patient location } \\
\hline & & & & & & ICU (N\%) & Wards (N\%) & OP (N\%) \\
\hline \multirow[t]{3}{*}{$\begin{array}{l}\text { Candida } \\
\text { non } \\
\text { albicans }\end{array}$} & $\begin{array}{l}\text { Candida Spp } \\
\text { (C. glabrata, } \\
\text { C. kefyr, C. } \\
\text { parapsilosis, } \\
\text { C. Iusitaniae) }\end{array}$ & 180 & 112 & 100 & 62.2 & $152(84.4 \%)$ & $27(15 \%)$ & $1(0.5 \%)$ \\
\hline & C. krusei & & 43 & & 23.8 & & & \\
\hline & C. tropicalis & & 25 & & 13.8 & & & \\
\hline
\end{tabular}

Isolates of non-albicans Candida were collected from different patient population in different wards. Most of candida isolates were collected from ICU wards $83.7 \%$ followed by in patient's wards $15.4 \%$, then $0.8 \%$ from outpatient samples as shown in Table 2.

Classification of sample types of Candida nonalbicans was as follows; candiduria represents $89.4 \%$, followed by candidemia 5.6, then Candida infection in wounds $4 \%$ while the least type of samples was candida isolated from ear 0.8 .

After subjecting all isolates $\mathrm{n}=180$ to high temperature at $42^{\circ} \mathrm{C}, 170(94.4 \%)$ showed no growth, while only $10(5.6 \%)$ isolates were grown at $42^{\circ}$. The 10 isolates were cultured from urine samples (candiduria) and were identified by Chromagar as 90\% Candida species (C. glabrata, C. kefyr, C. parapsilosis, and C. Iusitaniae) and $10 \%$ C. krusei.

The accurate identification of thermotolerant isolates was done using MALDI-TOF. Identification of the organism being tested is automatically determined using software that compares the spectral profile of the unknown organism with a reference database. Five of them were identified as C. glabrata, four were identified as C. krusei, and one was identified as C. kefyr.

The antimicrobial susceptibility of MALDITOF identified isolates to three antifungals using $E$ test is shown in Table 3; caspofungin and amphotericin were highly effective while flicin were highly effective while ALDI-TOF isolates. The lowest MIC values were recorded for amphotericin B with C. krusei with a susceptible range of $0.01-0.1$; for caspofungin with C. glabrata, with a susceptible range of 0.0-0.02; and C. kefyr with amphotericin 0.02 .

Table 3: Antimicrobial resistance of thermo-tolerant isolates

\begin{tabular}{|c|c|c|c|}
\hline Identified isolate & Antifungal & MIC values (ug/ml) & Interpretation \\
\hline \multirow[t]{3}{*}{ C. glabrata $(n=5)$} & Amphoteracin & $0.02-0.3$ & Sensitive \\
\hline & Fluconazole & 48-64 & Resistant \\
\hline & Caspofungin & $0.0-0.2$ & Sensitive \\
\hline \multirow[t]{2}{*}{${ }^{*} \mathrm{C}$. krusei $(\mathrm{n}=4)$} & Amphoteracin & $0.01-0.1$ & Sensitive \\
\hline & Caspofungin & $0.01-0.6$ & Sensitive \\
\hline \multirow[t]{3}{*}{ C. kefyr (other species) $(n=1)$} & Amphoteracin & 0.02 & Sensitive \\
\hline & Fluconazole & 16 & Resistant \\
\hline & Caspofungin & 0.15 & Sensitive \\
\hline
\end{tabular}

After excluding the four intrinsically resistant $C$. krusei isolates, fluconazole resistance was recorded in $100 \%$ of other non-albicans isolates.

The MICs for fluconazole, caspofungin, and amphotericin B for C. parapsilosis ATCC 22019 ranged from 1 to $3 \mu \mathrm{g} / \mathrm{mL}$, from 0.3 to $0.5 \mu \mathrm{g} / \mathrm{mL}$, and from 0.6 to $1 \mu \mathrm{g} / \mathrm{mL}$, respectively; the results met expected ranges according to CLSI, as shown in Figure 1 [19].

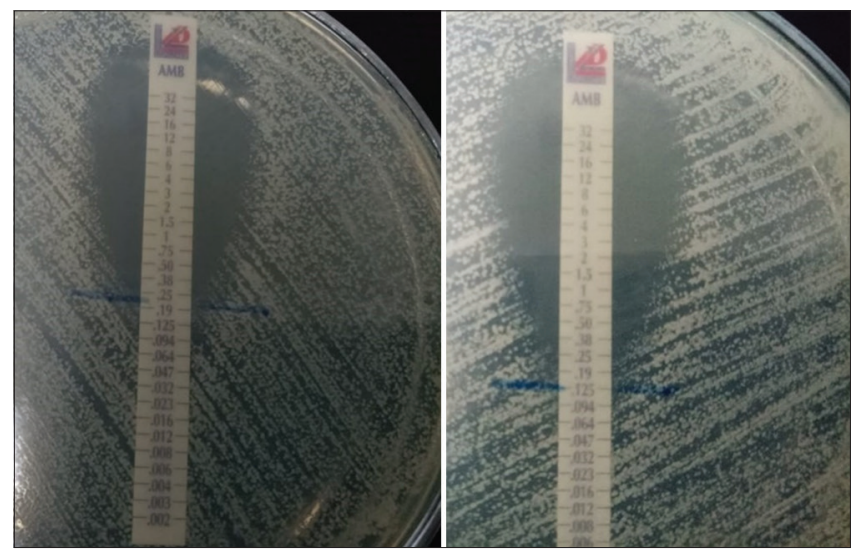

Figure 1: Amphotericin E test result of two isolates of Candida glabrata

\section{Discussion}

C. auris is a newly emerged Candida species which presents a serious health threat and outbreaks in health-care settings. Isolates are resistant to at 
least one antifungal drug; in addition, it could not be identified easily by standard laboratory methods [2]. Consequently, it is crucial to detect the prevalence in each country, and setting a protocol for rapid and accurate diagnosis of $C$. auris.

In this study, 400 clinical candida isolates were collected to detect the prevalence of $C$. auris strains among candida isolates in Cairo University Hospitals from February 2018 to December 2019 using a set of sequential steps for identification based on CDC guidelines.

Our study showed that 227 isolates (56.75\%) were C. albicans and 180 (43.25\%) were non-albicans Candida as detected by germ tube and confirmed by Chromagar. Non-albicans Candida were isolated at high frequency from patients and this is in agreement with Ferreira et al. who stated that Candida species are the most frequent fungi isolated from hospitals; this is in contrast to few years ago where $C$. albicans was the predominant organism in health-care settings [20]. Different non-albicans species were isolated by Chromagar ranging from $25(13.8 \%)$ C. tropicalis, $43(23.8 \%)$ were C. krusei, and $112(62.2 \%)$ were other Candida species (C. glabrata, C. kefyr, C. parapsilosis, and C. lusitaniae). Most of non-albicans Candida $84.4 \%$ were collected from ICUs, while $15 \%$ from inpatients wards. This indicates the prevalence of non-albicans Candida and its pathogenicity in ICU patients which could be explained by the frequent usage of antibiotics, and indwelling devices, especially in immunecompromised and severely ill patients as stated by Deorukhkar et al. [21].

The most common specimen for non-albicans Candida isolated in this study was urine $(89.4 \%)$, followed by candidemia (5.6\%), then wounds (4\%). This was in accordance with Singla et al. [22] who declared that candiduria is the most common specimens for nonalbicans Candida, especially in ICUs; however, urine specimens represents the least to isolate Candida in healthy individuals [23].

After segregating Candida isolates using two methods for confirmation, we followed the set steps for identification, the 180 isolates of non-albicans were subjected to high temperature at $42^{\circ} \mathrm{C}$. Phenotypical characteristics showed that most Candida species are unable to grow at high temperature with exception to few including $C$. auris [24]. Ten isolates of the 180 were thermotolerant and grew at $42^{\circ} \mathrm{C}$ and were suspected to be $C$. auris. For accurate identification according to $C D C$, MALDI-TOF was used for the definite identification, five isolates were identified as C. glabrata, and four isolates were identified as $C$. krusei and one $C$. kefyr. This result was in accordance with Bezjak and Chandy who stated that species of C. glabrata, C. krusei, and C. kefyr can grow at high temperature as C. auris [25].

Antimicrobial resistance of the 10 isolates showed high efficacy of amphotericin B and echinocadins, but high resistance against fluconazole this was in agreement with Berkow and Lockhart [26] who detected the high resistance of fluconazole among Candida nonalbicans isolates. The discrepancy shown between the identification by Chromagar, the most commonly used method for Candida identification, and MALDI-TOF was alarming. Nine of the thermotolerant isolates were detected as Candida species (C. glabrata, C. kefyr, C. parapsilosis, and C. lusitaniae) while one isolate detected as $C$. krusei by Chromagar; in contrast, MALDITOF detected five isolates as C. glabrata, four isolates as C. krusei, and one C. kefyr. Missed identification of C. krusei by Chromagar represents a pit fall in diagnosis and selection of treatment options for patients as $C$. krusei is intrinsic resistant to fluconazole. This defect in phenotypic identification highlights the importance of MALDI-TOF in rapid and accurate identification of fungal isolates, especially in ICU patients. This study detected that $C$. auris is not prevalent in Egypt and confirmed the reliability of the set protocol for identification and this was in concordance with CDC case tracking in January 2021 which tracked 1625 cases worldwide including only one isolate reported from Egypt [27].

These findings also confirmed by Lu et al., 2018, who conducted a detailed search for $C$. auris in Taiwan of more than 5000 archived Candida isolates from the period 1999 to 2016 and no isolates detected [28]. Moreover, Lockhart et al., 2017, looked for misidentified or overlooked candida before the recent emergence, they queried the international surveillance program SENTRY which contained 15,271 Candida isolates collected from 2004 to 2015 . Four isolates only were identified as $C$. auris supporting its scarcity which is in accordance with our findings [29].

However, despite scarcity of $C$. auris isolates [30], clinical microbiologists should be aware and have a definite protocol for prevention, prompt, and early identification due to the high resistance and aggressiveness of this bug.

\section{Conclusion and Limitation of Work}

It is concluded from this study that $C$. auris is not prevalent in Egyptian hospitals. In addition, Chromagar could be used as a screening method; however, definite diagnosis of isolate species for follow-up of treatment requires a more advanced technique, such as MALDI-TOF. The strength of this study is in the number of isolates collected where it reflects a wide sampling range of Candida organisms. One limitation is the limited number of hospitals from where Candida was collected. Isolates were collected from three Cairo University Hospitals; however, larger number of hospitals is preferred in further studies to reflect a bigger image of the situation in Egypt. 


\section{References}

1. Brown GD, Denning DW, Gow NA, Levitz SM, Netea MG, White TC. Hidden killers: Human fungal infections. Sc Transl Med. 2012;4(165):165rv13. https://doi.org/10.1126/ scitransImed.3004404

PMid:23253612

2. Du H, Bing J, Hu T, Ennis CL, Nobile CJ, Huang G. Candida auris: Epidemiology, biology, antifungal resistance, and virulence. PLoS Pathog. 2020;16(10):e1008921. https://doi. org/10.1371/journal.ppat.1008921

PMid:33091071

3. Centers for Disease Control and Prevention. CDC Tracking Candida auris. Atlanta, GA, USA: Centers for Disease Control and Prevention; 2020. Available from: https://www.cdc.gov/ fungal/candida-auris/tracking-c-auris.html. [Last accessed on 2020 Dec 01]. https://doi.org/10.1093/ofid/ofv131.60

4. Chen J, Tian S, Han X, Chu Y, Wang Q, Zhou B, Shang H. Is the superbug fungus really so scary? A systematic review and meta-analysis of global epidemiology and mortality of Candida auris. BMC Infect Dis. 2020;20(1):1-10. https://doi.org/10.1186/ s12879-020-05543-0

5. Sabino R, Veríssimo C, Pereira ÁA, Antunes F. Candida auris, an agent of hospital-associated outbreaks: Which challenging issues do we need to have in mind? Microorganisms. 2020;8(2):181. https://doi.org/10.3390/microorganisms8020181 PMid:32012865

6. Centers for Disease Control and Prevention (CDC). Recommendations for Infection Prevention and Control for Candida auris, Fungal Diseases, Candida auris, CDC; 2018. Available from: https://www.cdc.gov/fungal/candida-auris/cauris-infection-control.html. [Last accessed on 2017 Sep 14]. https://doi.org/10.34297/ajbsr.2019.03.000625

7. Rybak JM, Muñoz JF, Barker KS, Parker JE, Esquivel BD, Berkow EL, et al. Mutations in TAC1B: A novel genetic determinant of clinical fluconazole resistance in Candida auris. mBio. 2020;11(3):e00365-20. https://doi.org/10.1128/ mbio.00365-20

PMid:32398311

8. Arastehfar A, Shaban T, Zarrinfar H, Roudbary M, Ghazanfari M, Hedayati MT, et al. Candidemia among Iranian patients with severe COVID-19 admitted to ICUs. J Fungi (Basel). 2021;7(4):280. https://doi.org/10.3390/jof7040280

PMid:33917967

9. Chakrabarti A, Sood P. On the emergence, spread and resistance of Candida auris: Host, pathogen and environmental tipping points. J Med Microbiol. 2021;70(3):001318. https://doi. org/10.1099/jmm.0.001318

PMid:33599604

10. Kmeid J, Jabbour JF, Kanj SS. Epidemiology and burden of invasive fungal infections in the countries of the Arab League. J Infect Public Health. 2020;13(12):2080-6. https://doi. org/10.1016/j.jiph.2019.05.007

11. Centers for Disease Control and Prevention(CDC), National Center for Emerging and Zoonotic Infectious Diseases (NCEZID), Division of Foodborne, Waterborne, and Environmental Diseases (DFWED). Recommendations for Identification of Candida auris, Fungal Diseases, Candida auris, CDC; 2018. Available from: https://www.cdc.gov/fungal/candidaauris/recommendations.html\#suspect. [Last accessed on 2018 Oct 04]. https://doi.org/10.1016/s1473-3099(18)30609-1

12. BioMérieux Product List; 2012. Available from: https://www. biomerieux-diagnostics.com/conventional-media-fungalinfections. [Last accessed on 2018 Jul???].

13. Sarma S, Upadhyay S. Current perspective on emergence, diagnosis and drug resistance in Candida auris. Infect Drug Resist. 2017;10:155-65. https://doi.org/10.2147/idr.s116229 PMid:28652784

14. Moya-Salazar J, Rojas R. Comparative study for identification of Candida albicans with germ tube test in human serum and plasma. Clin Microbiol Infect Dis. 2018;3(3):1-4. https://doi. org $/ 10.15761 / \mathrm{cmid} .1000143$

15. Oxoid Product List; 2012. Available from: http://www.oxoid.com/ uk/blue/prod_detail/prod_detail.asp?pr $=\mathrm{cm} 1002$ and cat $=$ and $\mathrm{c}=\mathrm{uk}$ and lang=en. [Last accessed on 2019 Jul???].

16. Alastruey-Izquierdo A, Melhem MS, Bonfietti LX, RodriguezTudela JL. Susceptibility test for fungi: Clinical and laboratorial correlations in medical mycology. Rev Inst Med Trop São Paulo. 2015;57 Suppl 19:57-64. https://doi.org/10.1590/ s0036-46652015000700011

PMid:26465371

17. Clinical and Laboratory Standards Institute. Performance Standards for Antifungal Susceptibility Testing of Yeasts; Approved, Standard. CLSI document M60. $1^{\text {st }}$ ed. Wayne, PA: Clinical and Laboratory Standards Institute; 2017. p. 26. https:// doi.org/10.1007/978-1-59745-134-5_2

18. Lass-Flörl C, Arendrup MC, Rodriguez-Tudela JL, CuencaEstrella M, Donnelly P, Hope W, et al. EUCAST technical note on amphotericin B. Clin Microbiol Infect. 2011;17(12):E27-9. https://doi.org/10.1111/j.1469-0691.2011.03644.x

PMid:22011310

19. Barry AL, Pfaller MA, Brown SD, Espinel-Ingroff A, Ghannoum MA, Knapp C, et al. Quality control limits for broth microdilution susceptibility tests of ten antifungal agents. J Clin Microbiol. 2000;38(9):3457-9. https://doi.org/10.1128/ jcm.38.9.3457-3459.2000

PMid:10970403

20. Ferreira AV, Prado CG, Carvalho RR, Dias KS, Dias AL. Candida albicans and Non-C. Albicans Candida species: Comparison of biofilm production and metabolic activity in biofilms, and putative virulence properties of isolates from hospital environments and infections. Mycopathologia. 2013;175(3-4):265-72. https://doi. org/10.1007/s11046-013-9638-z

PMid:23532754

21. Deorukhkar SC, Saini S, Mathew S. Non-albicans Candida infection: An emerging threat. Interdiscip Perspect Infect Dis. 2014;2014:615958.

PMid:25404942

22. Singla N, Gulati N, Kaistha N, Chander J. Candida colonization in urine samples of ICU patients: Determination of etiology, antifungal susceptibility testing and evaluation of associated risk factors. Mycopathologia. 2012;174(2):149-55. https://doi. org/10.1007/s11046-011-9514-7 PMid:22723047

23. Sobel JD, Fisher JF, Kauffman CA, Newman CA. Candida urinary tract infections-epidemiology. Clin Infect Dis. 2011;52 Suppl 6:S433-6. https://doi.org/10.1093/cid/cir109 PMid:21498836

24. Oh BJ, Shin JH, Kim MN, Sung H, Lee K, Joo MY, et al. Biofilm formation and genotyping of Candida haemulonii, Candida pseudohaemulonii, and a proposed new species (Candida auris) isolates from Korea. Med Mycol. 2011;49(1):98-102. https://doi.org/10.3109/13693786.2010.493563 PMid:20560864

25. Bezjak V, Chandy R. Growth of fresh human isolates of some Candida species at $45^{\circ}$ C. J Med Vet Mycol. 1989;27(3):197200. https://doi.org/10.1080/02681218980000271 PMid:2674386

26. Berkow EL, Lockhart SR. Fluconazole resistance in Candida species: A current perspective. Infect Drug Resist. 
2017;10:237-45. https://doi.org/10.2147/idr.s118892 PMid:28814889

27. Tracking Candida auris, CDC; 2021. Available from: https:// www.cdc.gov/fungal/candida-auris/tracking-c-auris.html. [Last accessed on 2021 Jan 13].

28. Lu PL, Liu WL, Lo HJ, Wang FD, Ko WC, Hsueh PR, et al. Are we ready for the global emergence of multidrug-resistant Candida auris in Taiwan? J Formos Med Assoc. 2018;117(6):462-70. https://doi.org/10.1016/j.jfma.2017.10.005 PMid:29122404

29. Lockhart SR, Etienne KA, Vallabhaneni S, Faroogi J,
Chowdhary A, Govender NP, et al. Simultaneous emergence of multidrug-resistant Candida auris on 3 continents confirmed by whole-genome sequencing and epidemiological analyses. Clin Infect Dis. 2017;64(2):134-40. https://doi.org/10.341 0/f.727142249.793538208

\section{PMid:27988485}

30. Abastabar M, Haghani I, Ahangarkani F, Rezai MS, Armaki MT, Roodgari S, et al. Candida auris otomycosis in Iran and review of recent literature. Mycoses. 2019;62(2):101-5. https://doi. org $/ 10.1111 /$ myc. 12886

PMid:30585653

\section{Author Queries???}

AQ4: Kindly provide last accessed date 